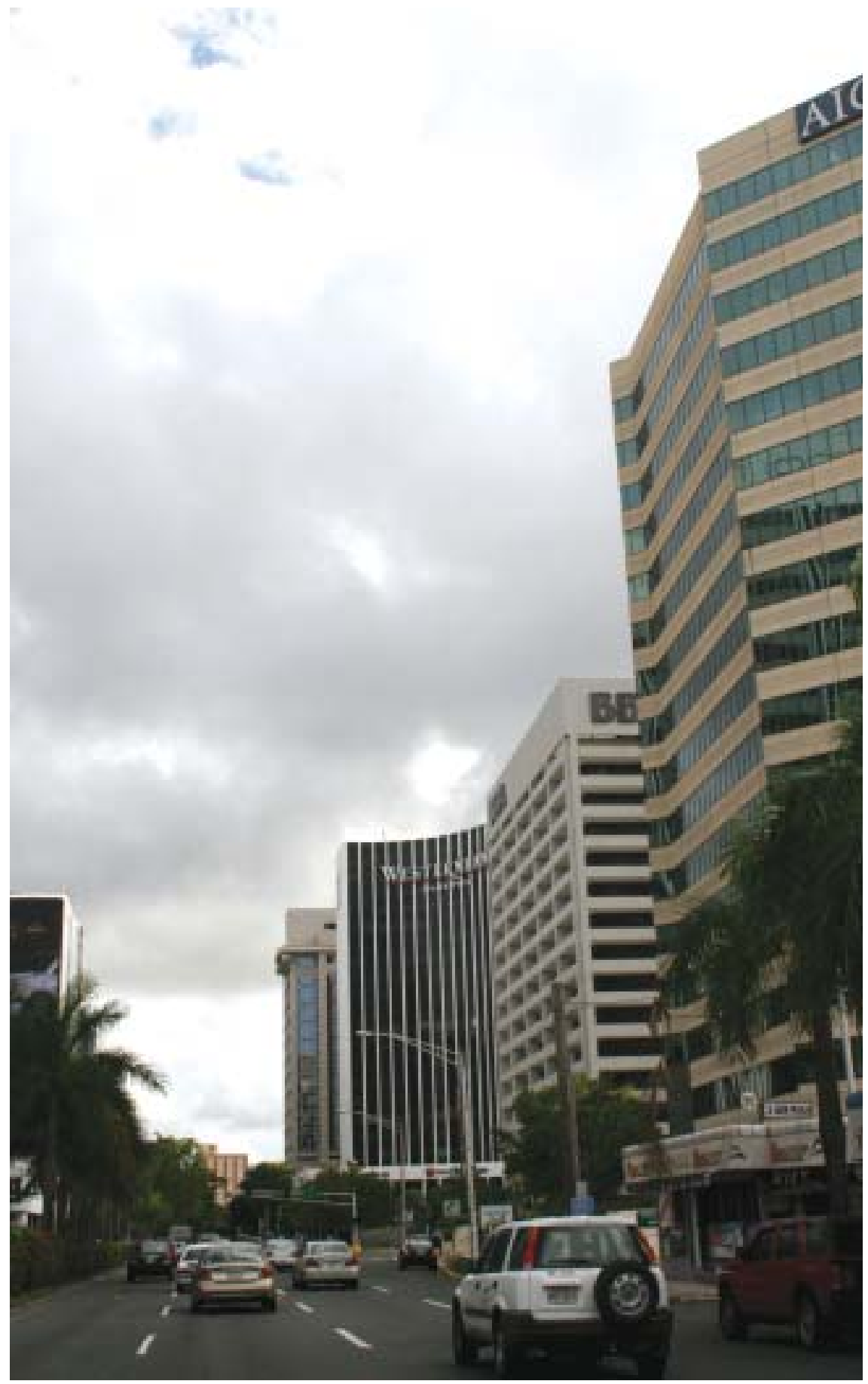




\title{
Resumen
}

En este trabajo se analizan las actitudes hacia la creación de empresas de dos muestras de estudiantes universitarios de Catalunya y Puerto Rico, respectivamente. Se estudian las percepciones de deseabilidad, viabilidad e intencionalidad de crear empresaspropias. El marco teórico de referencia utilizado es la Teoría Económica Institucional, dada su adecuación para el estudio de los factores que condicionan la actividad empresarial. Excepto diferencias poco relevantes, los resultados obtenidos en cuanto a las actitudes hacia la creación de empresas son muy similares para las dos muestras consideradas.

Palabras Clave: creación de empresas, deseo, viabilidad, intención, actitudes, teoría económica institucional, Cataluña, Puerto Rico.

\begin{abstract}
In this research the attitudes towards entrepreneurship of two samples of university students from Catalonia and Puerto Rico are analysed. Perceptions of desirability, feasibility and the intention to start a new firm are studied and compared. Institutional Economic Theory is used as a theoretical framework of the research due to its adequacy to the analysis of the conditioning factors to entrepreneurship. Not relevant differences in the attitudes to entrepreneurship between Catalonia and Puerto Rico are found in the research.
\end{abstract}

Key Words: entrepreneurship, desirability, feasibility, intention attitudes, Institutional Economic Theory, Catalonia, Puerto Rico. 


$$
\begin{gathered}
\text { M arinés A ponte* } \\
\text { David U rbano** } \\
\text { José M. Veciana*** }
\end{gathered}
$$

\section{Actitudes hacia la creación de empresas: Un estudio comparativo entre Catalunya y Puerto Rico}

*U niversidad de Puerto Rico, Facultad de Empresas, Recinto de Río Piedras

**Universidad Autónoma de Barcelona, Facultad de Ciencias Económicas yEmpresariales, Barcelona España

***U niversidad Autónoma de Barcelona, Facultad de Ciencias Económicas y Empresariales Barcelona, España

Recibido: 10 de octubre de 2005

Aceptado: 11 de septiembre de 2006

Disponible en línea: 11 de diciembre de 2006

\section{Introducción}

n las últimas décadas, la teoría económica institucional ha destacado la importancia de los factores institucionales o del entorno como determinantes del espíritu empresarial y la actividad emprendedora. Actualmente, se considera que las instituciones constituyen, en cierta medida, las reglas de juego de una sociedad. En este sentido, el contexto institucional condiciona el funcionamiento y el desempeño de las economías ( North, 1990:3). Éste entiende 
que institución es cualquier tipo de limitación o norma que las personas diseñan en la construcción de la interacción humana. Éstas pueden ser instituciones formales tales como las reglamentaciones políticas, legislación económica y contratos, o informales tales como los valores, actitudes, códigoso normas de comportamiento y convicciones sociales.

Varios autores emplean la teoría económica institucional con propósitossimilares en distintos contextos ${ }^{1}$. Entre ellos, se destaca Veciana (1999: 25) quien plantea que: "[ ... ] la teoría institucional es, sin duda, la teoría que actualmente proporciona un marco conceptual más consistente y apropiado para el estudio de la influencia de los factores del entorno en la función empresarial y la creación de empresas".

El contexto social y cultural -valores, actitudes y hábitos- condiciona, en alto grado, las decisiones que inducen a determinados comportamientos. En el caso de la creación de empresas, las percepciones de deseabilidad y viabilidad son producto del entorno cultural y social y determinan cuáles acciones o comportamientos serán considerados y al final adoptados (Shapero, 1982).

El propósito principal del presente trabajo es identificar las actitudes y creencias hacia la creación de empresas de una muestra de estudiantes universitarios de Catalunya y Puerto Rico. La selección de estos dos países como base comparativa responde a las semejanzas culturales que existen entre ambos, fruto de una tradición latina común. A la vez, dicha comparación se consideró interesante, dadas las diferencias entre los modelos económicos que predominan en estos dos países. Mientras que Catalunya fundamenta su modelo en las PYMEs, Puerto Rico se basa en los incentivos fiscales de atracción de capital extranjero, concretamente de los Estados U nidos de América.

La investigación pretende dar respuesta a las siguientes interrogantes':

1) ¿Cuál es la motivación para crear una empresa propia por parte de los estudiantes universitarios?

2) ¿Tienen los estudiantes universitarios una percepción positiva o negativa sobre la viabilidad de crear una nueva empresa?

3) Dichos estudiantes, ¿tienen la intención de crear una nueva empresa en el futuro? 
El cuestionario que se utilizó en esta investigación se basa en el empleado en un estudio realizado en España (Genescá y Veciana, 1984), complementado con preguntas adicionales en torno a la percepción sobre la viabilidad de la nueva empresa. Se contrastaron seis hipótesis relacionadas con aspectos destacados en la literatura y desarrollados en el marco teórico, según se explica en la próxima sección. El artículo presenta un análisis comparativo entre los dos países considerados.

Los resultados de la presente investigación son importantes, al menos, por dos razones. Por un lado, si las actitudes hacia la actividad emprendedora son consideradas como producto del entorno social y cultural, un conocimiento más profundo sobre el deseo, la viabilidad y la intención de crear una empresa puede servir de base para el diseño de políticas gubernamentales destinadas a mejorar dicho entorno institucional. Por otro, los estudiantes universitarios representan uno de los colectivos claves susceptibles para convertirse en empresarios ( Veciana, 1998b). En este sentido, el mejor conocimiento sobre sus actitudes hacia la actividad empresarial puede significar el primer paso para despertar y estimular el interés de los estudiantes hacia una carrera como empresario independiente.

\section{Marco teórico e hipótesis de investigación}

Krueger y Brazeal ( 1994) desarrollaron un modelo sobre el potencial emprendedor de la persona, que se ajusta a los planteamientos de la teoría económica institucional. El modelo ha sido integrado al análisis en esta investigación. Éste tiene una perspectiva psico-social basada en el concepto sobre proceso que tiene consecuencias a nivel macro (Krueger y Brazeal, 1994:91). Dicho modelo se apoya en el trabajo de Áspero ( 1982) sobre el suceso disparador y en la teoría del comportamiento planificado de Ajzen( 1991).

El modelo de Shapero sobre la formación del evento empresarial parte de cómo el entorno social afecta la elección de la carrera empresarial. Dicho autor afirma que la intención de crear una nueva empresa se deriva de las percepciones de deseabilidad y viabilidad. Por otra parte, la teoría del comportamiento planificado de Ajzen (1991) que pretende explicar y predecir el comportamiento humano, 
se centra en la intención de un individuo de actuar de una manera determinada. El modelo de Krueger y Brazeal sobre el potencial emprendedor sitúa al trabajo de Shapero en el contexto del proceso intencional. Entendemos que la teoría del comportamiento planificado de Ajzen yel modelo de Shapero sobre la creación de empresas se solapan según se muestra a continuación en la Tabla 1.

Tabla 1

Equivalencias entre los modelos

\begin{tabular}{|l|l|}
\hline \multicolumn{1}{|c|}{ Modelo de Shapero } & \multicolumn{1}{c|}{ Teoría de Ajzen } \\
\cline { 1 - 1 } Percepción sobre el deseo de crear una empresa & - Actitud hacia el comportamiento \\
Percepción sobre la viabilidad de crear una empresa & - Norma subjetiva \\
\hline
\end{tabular}

A partir de la terminología de Shapero, el modelo de Krueger y Brazeal integra los conceptos de percepción, deseabilidad y viabilidad según se muestra en la Figura 1.

\section{Diagrama 1 \\ Modelo del potencial emprendedor}

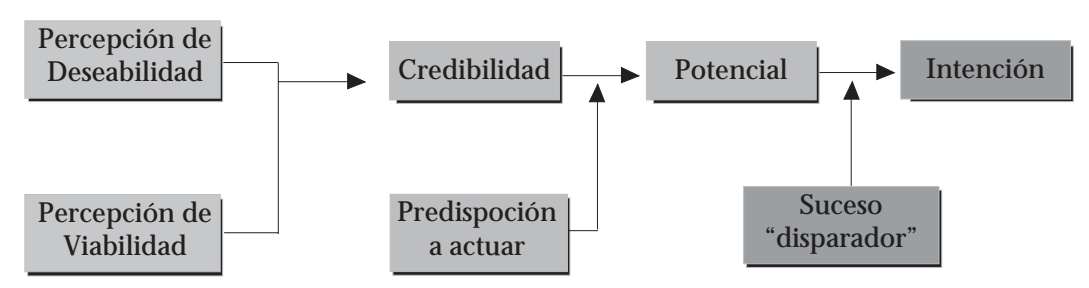

Fuente: Krueger y Brazeal (1994:95)

Como se puede ver en el Diagrama 1, la credibilidad del evento requiere que el comportamiento se considere deseable y viable. Estos antecedentes afectan las intenciones del comportamiento o acción para crear una nueva empresa. El modelo también explica, que aunque el individuo puede percibir que la creación de una nueva empresa es deseable y viable y, por tanto verosímil, dicho individuo 
puede que no se decida implantar el comportamiento debido a la falta del suceso disparador.

Por otro lado, según se ilustra en el Diagrama 2, la teoría sobre el comportamiento planificado postula que el comportamiento es una función de las creencias sobre una determinada conducta. Se considera que estas creencias son los determinantes principales de las actitudes, intenciones y el comportamiento de una persona (Ajzen, 1991:189).

\section{Diagrama 2}

El comportamiento como función de las creencias

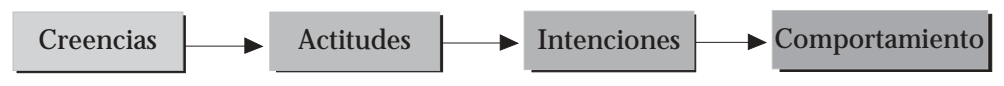

Fuente: Ajzen (1991:189)

Asimismo, Shapero ( 1982) examina el concepto de deseabilidad usando información sobre la familia, círculos de amistad, grupos étnicos y entorno educativo y profesional de los empresarios potenciales. En el caso de la familia, afirma dicho autor, que particularmente el padre o la madre desempeñan un papel muyimportante en la consideración de la deseabilidad y credibilidad sobre la función empresarial por parte del futuro empresario.

En cuanto a la percepción sobre la viabilidad, la teoría del comportamiento planificado plantea que los recursos y oportunidades que tiene una persona conducen a un determinado comportamiento. En este sentido, Shapero ( 1982) subraya que la percepción sobre la disponibilidad de recursos o apoyo financiero puede hacer que el empresario potencial perciba como más viable la creación de una empresa propia, haciendo más factible este suceso.

En la misma línea, Krueger (1993) destaca que, mientras que la percepción sobre la credibilidad, el deseo y la propensión a actuar explican algo más del cincuenta por ciento de la varianza de las intenciones de crear una empresa, la percepción de viabilidad explica la mayor parte de la misma. 


\section{H ipótesis de investigación}

La literatura sobre la creación de empresas que estudia la relación entre los antecedentes de empresarios en la familia y el género del empresario con la creación de nuevas empresas es muy amplia. Por un lado, los trabajos en esta línea plantean una relación positiva entre la presencia de antecedentes empresariales en la familia y la decisión de los empresarios potenciales de iniciar una nueva empresa. Collins, Moore y Unwalla (1964) fueron los primeros autores en demostrar, por medio de un estudio empírico, que las influencias sobre la conducta empresarial se remontan a la niñez y a las circunstancias familiares del empresario (Veciana, 1988:14) ${ }^{1}$.

Por otro lado, algunos de los trabajos que analizan la relación entre el género ylasactitudes hacia la creación de empresas (Scherer et al., 1989; M atthews y M oser, 1995; Kolvereid, 1996; Kourilsky y Walstad, 1998), han evidenciado que los hombres tienen mayor tendencia que las mujeres hacia la conducta empresarial (Scherer et al., 1989; Matthews y Moser, 1995).

De todas formas, cabe decir que los modelos demográficos utilizados en las investigaciones anteriormente citadas han sido criticados debido a que aportan poco en torno a cómo las variables de familiares empresarios e influencias sociales afectan el proceso decisional del individuo en relación con la conducta empresarial (Katz, 1992). En este sentido, modelos como el de Krueger y Brazeal que se centran en las intenciones como predictores del comportamiento empresarial se han sugerido como alternativas a los modelos demográficos.

Kolvereid ( 1996) plantea que los modelos demográficos han aportado resultados empíricos que dan apoyo a las hipótesis que relacionan los antecedentes familiares y el género, con las intenciones empresariales ( Mathews y M oser, 1995). A pesar de ello, Robinson et al. (1991) argumentan que no existe una relación directa entre las variables demográficas y el comportamiento empresarial. Scherer et al. (1989) yKrueger (1993) plantean que los ejemplos sobre empresarios con éxito pueden condicionar la intención de crear una empresa, pero sólo en el caso de que éstos afecten las actitudes. 
Kolvereid (1996) aplicó la teoría sobre el comportamiento planificado para predecir cómo las personas eligen la carrera empresarial, relacionando esta decisión con el papel de la familia, el género y la experiencia profesional previa. Los resultados más relevantes de su estudio indican que los hombres muestran una mayor preferencia por el autoempleo que las mujeres. Asimismo, la relación entre los antecedentes empresariales en la familia con la intención de crear una empresa propia apareció como positiva, aunque no estadísticamente significativa.

Las hipótesis que contrastamos en el presente trabajo responden al cruce de las variables demográficas-ampliamente estudiadas en la literatura sobre la creación de las empresas- con los aspectos más relevantes del modelo de Krueger y Brazeal. Así, se cruzaron las variables de género y antecedentes de empresarios en la familia con el deseo, viabilidad e intención de crear una nueva empresa. A continuación se presentan las seis hipótesis que se consideraron:

H 1: No existe relación de dependencia entre el género de los estudiantes y la percepción de deseo de crear una nueva empresa.

H 2: No existe relación de dependencia entre el género de los estudiantes y la percepción de viabilidad de crear una nueva empresa.

H 3: No existe relación de dependencia entre el género de los estudiantes y la firme intención de crear una nueva empresa.

H4: Existe una relación de dependencia entre la variable antecedentes de empresarios en la familia y la percepción de deseo de crear una nueva empresa.

H 5: Existe una relación de dependencia entre la variable antecedentes de empresarios en la familia y la percepción de viabilidad de crear una nueva empresa.

H6: Existe una relación de dependencia entre la variable antecedentes de empresarios en la familia y la firme intención de crear una nueva empresa. 


\section{Metodología de la investigación}

\section{Métodos para el recogido de la información, ficha técnica de la investigación y métodos de análisis de datos}

Para el análisis de las actitudes de los estudiantes universitarios hacia la creación de nuevas empresas, se utilizó un cuestionario que se administró personalmente durante la sesión de clase a una muestra de 837 estudiantes universitarios en Catalunya, así como a una muestra de 435 en Puerto Rico.

La Tabla 2 presenta las características más relevantes de las muestras en la ficha técnica de la investigación:

\section{Tabla 2}

Ficha Técnica de la investigación: muestra de Catalunya y de Puerto Rico

\begin{tabular}{|c|c|c|}
\hline & CATALUNYA & PUERTO RICO \\
\hline Universo & $\begin{array}{c}\text { 18,641 estudiantes universitarios (de } 2^{\circ} \text { a } 4 \\
\text { o } 5^{\circ} \text { curso) matriculados en las titulaciones } \\
\text { de Administración de Empresas, } \\
\text { Economía, e Ingeniería de las } \\
\text { universidades UAB, UB, UPC, UdG, URV } \\
\text { y UdL, en el curso académico 1998-99. }\end{array}$ & $\begin{array}{l}15,000 \text { estudiantes universitarios (de } 2^{\circ} \text { a } 4^{\circ} \text { o } \\
5^{\circ} \text { curso) matriculados en las titulaciones } \\
\text { de Administración de Empresas, Economía, e } \\
\text { Ingeniería, de las universidades UPR, } \\
\text { Río Piedras, Mayagüez, Cayey; UIA y } \\
\text { Metro, en el curso académico 1998-99. }\end{array}$ \\
\hline $\begin{array}{l}\text { Ámbito } \\
\text { (universidades) }\end{array}$ & $\begin{array}{l}\text { Catalunya (UAB, UB, UPC, UdG, URV y } \\
\text { UdL) }\end{array}$ & $\begin{array}{l}\text { Puerto Rico (UPR, Río Piedras, Mayagüez, } \\
\text { Cayey; UIA y Metro) }\end{array}$ \\
\hline Tamaño muestral & $\begin{array}{c}837 \text { estudiantes universitarios matriculados } \\
\text { en las titulaciones de Administración de } \\
\text { Empresas, Economía e Ingeniería de las } \\
\text { universidades consideradas. }\end{array}$ & $\begin{array}{c}435 \text { estudiantes universitarios matriculados en } \\
\text { las titulaciones de Administración de Empresas } \\
\text { Economía e Ingeniería de las universidades } \\
\text { consideradas. }\end{array}$ \\
\hline Error muestral & $+/-3.31 \%$ & $+/-4.70 \%$ \\
\hline Nivel de confianza & $95 \% \mathrm{Z}=1.96 \mathrm{p}=\mathrm{q}=0.5$ & $95 \% \mathrm{Z}=1.96 \mathrm{p}=\mathrm{q}=0.5$ \\
\hline Diseño muestral & \begin{tabular}{|c|} 
Muestreo aleatorio estratificado \\
proporcional, considerando como variables \\
de \\
estratificación tanto la universidad de \\
procedencia, como la titulación cursada. \\
\end{tabular} & $\begin{array}{c}\text { Muestreo aleatorio estratificado proporcional, } \\
\text { considerando como variable de estratificación } \\
\text { la titulación cursada. }\end{array}$ \\
\hline Fecha trabajo campo & Entre diciembre 1999 y abril 2000 & Entre febrero y marzo de 1999 \\
\hline
\end{tabular}

En referencia al manejo de la información que se obtuvo a partir de la encuesta administrada a los estudiantes universitarios incluidos en la muestra, se utilizó el paquete estadístico SPSS Versión 11.5. Las técnicas estadísticasutilizadas fueron el análisisunivariable ( frecuencias 
y porcentajes) y el análisis bivariable (tablas de contingencia y contraste de medias para dos muestras).

Se aplicó la prueba de la Chi-Cuadrado $\left(\mathrm{x}^{2}\right)$ para medir las relaciones de dependencia entre las variables consideradas de interés y comprobar su significación estadística. En el análisis de comparación de medias se utilizó la prueba estadística t para comprobar la significación estadística de las diferencias grupales.

\section{Características de las muestras}

La muestra de Catalunya incluyó seis universidades públicas: Universitat Autònoma de Barcelona, Universitat de Barcelona, Universitat Politècnica de Catalunya, U niversitat de Lleida, Universitat de Girona y Universitat Roviri i Virgili. La distribución de los estudiantes encuestados por universidades en la muestra de Catalunya fue la siguiente: Universitat Autònoma de Barcelona, 27.2\%; Universitat de Barcelona, $15.5 \%$; U niversitat Politècnica de Catalunya, 13.0\%; Universitat de Lleida, 5.9\%; Universitat de Girona, 15.9\% y Universitat Roviri i Virgili, 22.1\%.

La muestra para Puerto Rico incluyó universidades públicas y privadas: Ia U niversidad de Puerto Rico, en Cayey, Mayagüez y Río Piedras; yla U niversidad Interamericana, Recinto Metropolitano. En este caso la distribución de los estudiantes encuestad os por universidades fue la siguiente: UPR-Cayey, 26.2\%; U PR-M ayagüez, 22.3\%; UPR-Río Piedras, 29.2\% e Interamericana-Metro, 22.3\%. En ambas muestras, la mayor parte de los estudiantes cursaba estudios en Administración y Dirección de Empresas, Economía e Ingeniería.

En cuanto a las características sociodemográficas, existen pocas diferencias entre las dos muestras. Por un lado, en la muestra catalana los estudiantes tienen en promedio una edad más el evada que los de la muestra de Puerto Rico. Éstos también se encuentran en cursos más avanzados ( $64 \%$ en 4ํaño académico vs. sólo 38.2\% en Puerto Rico). Por otro lado, el porcentaje de mujeres es mayor en Puerto Rico que en Catalunya ( $54.3 \%$ vs. $40.6 \%$ ). Por último, mientras que en Puerto Rico más de la mitad de la muestra ( $52.9 \%$ ) no tiene antecedentes de empresarios en la familia, sucede lo contrario para la muestra de Catalunya donde el 51.5\% afirmó tener empresarios en la familia. 


\section{Resultados y discusión}

En este apartado se presentan algunos de los resultados de la encuesta administrada a ambas muestras y se discuten los más relevantes. Además, se presenta el contraste de las hipótesis planteadas en la investigación.

\section{Percepción sobre el deseo y la viabilidad para crear una nueva empresa}

En la Tabla 4 se muestran los resultados que hacen referencia a la pregunta siguiente: ¿Considera usted deseable crear o fundar una empresa propia?

\section{Tabla 4}

Percepción de deseo y la viabilidad para crear una nueva empresa Por cientos

\begin{tabular}{|c|c|c|c|c|c|}
\hline \multicolumn{3}{|c|}{ Deseo } & \multicolumn{3}{|c|}{ Viabilidad } \\
\hline & $\begin{array}{c}\text { Catalunya } \\
(\%)\end{array}$ & $\begin{array}{c}\text { Puerto Rico } \\
(\%)\end{array}$ & & $\begin{array}{c}\text { Catalunya } \\
(\%)\end{array}$ & $\begin{array}{c}\text { Puerto Rico } \\
(\%)\end{array}$ \\
\hline Sí & 74.0 & 92.2 & Más fácil & 31.1 & 44.4 \\
\hline No & 25.6 & 7.8 & Más difícil & 66.1 & 53.5 \\
\hline No responde & e $\quad 0.4$ & ---- & No responde & 2.9 & 2.1 \\
\hline TOTAL & 100 & 100 & TOTAL & 100 & 100 \\
\hline
\end{tabular}

Según se observa en la Tabla 4, en ambos países la mayor parte de los estudiantes encuestados respondió afirmativamente a la pregunta, aunque la muestra de Puerto Rico cuenta con el porcentaje más elevado de respuestas afirmativas, $92.2 \%$ vs. $74 \%$ de Catalunya.

Si se comparan estos porcentajes con los del trabajo realizado por Genescà y Veciana ( 1984), se observa que la percepción de deseabilidad para crear una empresa ha mejorado de forma considerable en las dos últimas décadas, ya que para la investigación realizada en el año 1984 sólo el 56.8\% y el 66.7\%, para la muestra de España y Catalunya, respectivamente, respondieron afirmativamente a la pregunta. 
Por su parte, entre los estudiantes puertorriqueños que respondieron afirmativamente a dicha pregunta, el 50.4\% son hombres, el $57.3 \%$ tienen antecedentes de empresarios en la familia, y la mayoría (77.1\%) estaba cursando Dirección y Administración de Empresas. En el caso de Catalunya, el $61.3 \%$ de la muestra eran hombres, el $49.6 \%$ procedían de familias de empresarios, y también la mayoría (46.8\%) cursaba estudios en Dirección y Administración de Empresas.

Los resultad os relativosa la pregunta: ¿Considera usted que es más fácil o más difícil crear una empresa en la actualidad que en décadas anteriores?, se presentan en la Tabla 4.

En ambos países, la mayor parte de los estudiantes en las muestras respondió que es más difícil crear una empresa en la actualidad que en décadas anteriores, a pesar de que el porcentaje de respuestas fue más elevado en Catalunya (66.1\%) que en Puerto Rico, con $53.5 \%$.

Cabe decir que mientras los estudiantes puertorriqueños que respondieron más difícil eran mujeres (51.4\%), con antecedentes de empresarios en la familia (58.5\%) y se encontraban cursando los estudios de Administración de Empresas (80.7\%); los estudiantes catalanes eran mayoritariamente hombres (58.6\%), el $47.7 \%$ tenían empresarios en la familia y sólo el $42.9 \%$ estaban cursando Administración de Empresas.

A la luz de los resultados obtenidos, se concluye que la percepción sobre la viabilidad para crear una nueva empresa dependerá fundamentalmente de los factores que favorecen y de los factores que dificultan la fundación de empresas (Veciana, Aponte y U rbano, 1999).

\section{Intención de crear una nueva empresa}

En la Tabla 5 se presentan los resultados referentes a la pregunta: ¿H a pensado usted seriamente en crear o fundar una empresa propia? 
Tabla 5

Intención de crear una nueva empresa

Por cientos

\begin{tabular}{|lcc|}
\hline & $\begin{array}{c}\text { Catalunya } \\
(\mathbf{\%})\end{array}$ & $\begin{array}{c}\text { Puerto Rico } \\
\mathbf{( \% )}\end{array}$ \\
\hline No, nunca & 26.8 & 10.8 \\
No, pero pienso integrarme a una empresa de la familia & 4.2 & 2.1 \\
Sí, vagamente & 51.0 & 40.3 \\
Sí, seriamente & 12.1 & 28.7 \\
Sí, tengo el firme propósito de fundar una empresa & 4.1 & 16.1 \\
No responde & 1.8 & 2.0 \\
TOTAL & 100 & 100 \\
\hline
\end{tabular}

Según la tabla anterior, la mayor parte de las dos muestras de estudiantes respondió tener una vaga intención de crear su propia empresa, aunque este porcentaje es más elevado para la muestra catalana con $51.0 \%$ vs. $40.3 \%$ que para Puerto Rico. También el porcentaje de estudiantes que respondió No, nunca es mucho más elevado para la muestra catalana (26.8\%) que para la puertorriqueña (10.8\% ). De igual modo, para la muestra de Puerto Rico las respuestas Sí, seriamen te y Sí, tengo el firme propósito de fundar una empresa cuentan con porcentajes mucho más elevados que para Catalunya $28.7 \%$ y $16.1 \%$ y $12.1 \%$ y $4.1 \%$ respectivamente.

\section{Contraste de hipótesis}

Tal y como se comentó en la sección sobre la metodología, las relaciones de dependencia entre las variables demográficas, género y antecedentes de empresarios en la familia con el deseo, la viabilidad yla intención de crear una empresa se midieron mediante tablas de contingencia, aplicando la prueba Chi-Cuadrado $\left(x^{2}\right)$ para calcular su significación estadística y contrastar de esta manera las hipótesis de investigación.

H 1: No existe relación de dependencia entre el género de los estudiantes y la percepción de deseo de crear una nueva empresa.

La Tabla 6 muestra la prueba estadística para la H ipótesis 1. 
Tabla 6

Tabla de contingencia entre el género de los estudiantes y la percepción de deseo de crear una nueva empresa

Por cientos

\begin{tabular}{|lcccc|}
\hline & \multicolumn{2}{c}{ Catalunya (\%) } & \multicolumn{2}{c|}{ Puerto Rico (\%) } \\
\hline \multirow{2}{*}{ Masculino } & Sí & NO & SÍ & NO \\
Femenino & 76.9 & 23.1 & 95.7 & 4.3 \\
Deseo Total & 70.7 & 29.3 & 93.0 & 7.0 \\
PRUEBA ESTADISTICA & 74.4 & 25.6 & 94.4 & 5.6 \\
& \multicolumn{2}{c}{ Valor $\mathrm{x}^{2}=4.000^{* *}$} & \multicolumn{2}{c}{ Valor $\mathrm{x}^{2}=1.2054$} \\
\hline
\end{tabular}

$* *$ Sig $<0.05$

Mientras que para la muestra de Puerto Rico se acepta la H ipótesis 1, para la muestra de Catal unya se rechaza dicha hipótesis. En el caso de Catalunya existe una relación de dependencia entre el género de los estudiantes y la percepción de deseo de fundar una empresa. Dicha relación es estadísticamente significativa en un contraste " $\mathrm{x}^{2 \text { " }}$ al nivel de $95 \%$. El porcentaje de hombres que desean crear una empresa (76.9\%) es superior al de las mujeres (70.7\%), y además también es superior al porcentaje total de la muestra que había respondido afirmativamente a esta pregunta (74.4\%).

H2: No existe relación de dependencia entre el género de los estudiantes y la percepción de viabilidad de crear una nueva empresa.

La Tabla 7 muestra la prueba estadística para la H ipótesis N úmero 2.

\section{Tabla 7}

Tabla de contingencia entre el género de los estudiantes y la percepción de la viabilidad para crear una nueva empresa Por cientos

\begin{tabular}{|c|c|c|c|c|}
\hline & \multicolumn{2}{|c|}{ Puerto Rico (\%) } & \multicolumn{2}{|c|}{ Catalunya (\%) } \\
\hline & $\begin{array}{l}\text { Más fácil en la } \\
\text { actualidad que en } \\
\text { décadas anteriores }\end{array}$ & $\begin{array}{c}\text { Más difícil en la } \\
\text { actualidad que en } \\
\text { décadas anteriores }\end{array}$ & $\begin{array}{l}\text { Más fácil en la actualida } \\
\text { que en décadas anteriores }\end{array}$ & $\begin{array}{l}\text { Más difícil en la } \\
\text { actualidad que en } \\
\text { décadas anteriores }\end{array}$ \\
\hline Masculino & 48.8 & 51.2 & 32.4 & 67.6 \\
\hline Femenino & 49.4 & 50.6 & 31.5 & 68.5 \\
\hline Viabilidad Total & 49.1 & 50.9 & 32.1 & 67.9 \\
\hline PRUEBA ESTADÍSTICA & \multicolumn{2}{|c|}{ Valor $\mathrm{x}^{2}=0.1421$} & \multicolumn{2}{|c|}{ Valor $\mathrm{x}^{2}=0.72$} \\
\hline
\end{tabular}


La H ipótesis 2 se acepta para las dos muestras bajo estudio. En el caso de ambas muestras, no existe una relación de dependencia entre el género de los estudiantes y la percepción de la viabilidad para crear una empresa.

H 3: No existe relación de dependencia entre el género de los estudiantes y la firme intención de crear una nueva empresa.

La Tabla 8 presenta la prueba estadística para la H ipótesis 3. Esta H ipótesis se acepta para la muestra de Puerto Rico y se rechaza para la muestra de Catalunya. En la muestra de estudiantes catalanes existe una relación de dependencia entre el género y la intención de crear una empresa propia. Además, ésta es estadísticamente significativa en un contraste " $\mathrm{x}$ " al nivel de $95 \%$. El porcentaje de hombres que respondión Sí, seriamente ( $14.8 \%$ ) es más elevado que el porcentaje de mujeres ( $8.7 \%$ ) y que el porcentaje total de la muestra catalana (12.3\%) que respondió en este sentido.

\section{Tabla 8}

Tabla de contingencia entre el género de los estudiantes y la intención de crear una nueva empresa

\section{Por cientos}

\begin{tabular}{|c|c|c|c|c|c|}
\hline $\begin{array}{c}\text { CATALUNYA } \\
(\%)\end{array}$ & No, nunca & $\begin{array}{c}\text { No, pero pienso } \\
\text { integrarme a una } \\
\text { empresa de la familia }\end{array}$ & $\begin{array}{c}\text { Sí, } \\
\text { vagamente }\end{array}$ & $\begin{array}{c}\text { Sí, } \\
\text { seriamente }\end{array}$ & $\begin{array}{l}\text { Sí, tengo el firme } \\
\text { propósito de fundar } \\
\text { una empresa }\end{array}$ \\
\hline Masculino & 25.0 & 3.9 & 51.6 & 14.8 & 4.7 \\
\hline Femenino & 31.0 & 4.8 & 52.1 & 8.7 & 3.3 \\
\hline Intención Total & 27.4 & 4.3 & 51.8 & 12.3 & 4.1 \\
\hline PRUEBA ESTADÍSTICA & \multicolumn{5}{|c|}{ Valor $\mathrm{x}^{2}=9.764^{* *}$} \\
\hline $\begin{array}{c}\text { PUERTO RICO } \\
(\%)\end{array}$ & No, nunca & $\begin{array}{c}\text { No, pero pienso } \\
\text { integrarme a una } \\
\text { empresa de la familia }\end{array}$ & $\begin{array}{c}\text { Sí, } \\
\text { vagamente }\end{array}$ & $\begin{array}{c}\text { Sí, } \\
\text { seriamente }\end{array}$ & $\begin{array}{l}\text { Sí, tengo el firme } \\
\text { propósito de fundar } \\
\text { una empresa }\end{array}$ \\
\hline Masculino & 10.5 & 0.6 & 36.5 & 34.8 & 17.7 \\
\hline Femenino & 10.3 & 3.3 & 45.7 & 25.5 & 15.2 \\
\hline Intención Total & 10.4 & 1.9 & 41.1 & 30.1 & 16.4 \\
\hline PRUEBA ESTADÍSTICA & \multicolumn{5}{|c|}{ Valor $\mathrm{x}^{2}=8.30127$} \\
\hline
\end{tabular}

${ }^{* *} \operatorname{Sig}<0.05$

H 4: Existe una relación de dependencia entre la variable, antecedentes de empresarios en la familia y la percepción de deseo de crear una nueva empresa. 
La Tabla 9 muestra la prueba estadística para la H ipótesis N úmero 4.

Tabla 9

Tabla de contingencia entre los antecedentes de empresarios en la familia de los estudiantes y la percepción de deseo de crear una nueva empresa

Por cientos

\begin{tabular}{|llccc|}
\hline & \multicolumn{2}{c}{ Puerto Rico (\%) } & \multicolumn{2}{c|}{ CatalunyA (\%) } \\
& Sí & No & Sí & No \\
\hline Con antecedentes de empresarios en la familia & 95.8 & 4.2 & 75.9 & 24.1 \\
Sin antecedentes de empresarios en la familia & 93.3 & 6.7 & 72.7 & 27.3 \\
Deseo Total & 94.7 & 5.3 & 74.2 & 25.8 \\
PRUEBA ESTADÍSTICA & Valor $\mathrm{x}^{2}=1.15961$ & & \multicolumn{2}{c}{ Valor $\mathrm{x}^{2}=1.59$} \\
& \multicolumn{4}{r}{} \\
\hline
\end{tabular}

La H ipótesis 4 se rechaza para ambas muestras de estudiantes. No existe relación de dependencia entre el hecho de que los estudiantes tengan antecedentes de empresarios en sus respectivas familias y la percepción de crear una empresa propia.

H 5: Existe una relación de dependencia entre la variable antecedentes de empresarios en la familia y la percepción de viabilidad de crear una nueva empresa.

La Tabla 10 presenta la prueba estadística para la H ipótesis 5.

Tabla 10

Tabla de contingencia entre los antecedentes de empresarios en la familia de los estudiantes y la percepción de viabilidad de crear una nueva empresa Por cientos

\begin{tabular}{|c|c|c|c|c|}
\hline & \multicolumn{2}{|c|}{ Puerto Rico (\%) } & \multicolumn{2}{|c|}{ Catalunya (\%) } \\
\hline & $\begin{array}{l}\text { Más fácil en la } \\
\text { actualidad que en } \\
\text { décadas anteriores }\end{array}$ & $\begin{array}{l}\text { Más difícil en la } \\
\text { actualidad que en } \\
\text { décadas anteriores }\end{array}$ & $\begin{array}{l}\text { Más fácil en la } \\
\text { actualidad que en } \\
\text { décadas anteriores }\end{array}$ & $\begin{array}{l}\text { Más difícil en la } \\
\text { actualidad que en } \\
\text { décadas anteriore }\end{array}$ \\
\hline $\begin{array}{l}\text { Con antecedentes de empresarios } \\
\text { en la familia }\end{array}$ & 47.2 & 52.8 & 32.9 & 67.1 \\
\hline $\begin{array}{l}\text { Sin antecedentes de empresarios } \\
\text { en la familia }\end{array}$ & 52.3 & 47.7 & 31.3 & 68.7 \\
\hline Viabilidad Total & 49.4 & 50.6 & 32.1 & 67.9 \\
\hline PRUEBA ESTADÍSTICA & \multicolumn{2}{|c|}{ Valor $\mathrm{x}^{2}=0.8949$} & \multicolumn{2}{|c|}{ Valor $\mathrm{x}^{2}=0.251$} \\
\hline
\end{tabular}


Para ambas muestras se rechaza la Hipótesis 5, ya que no existe relación de dependencia entre los antecedentes de empresarios en la familia y la percepción de viabilidad para fundar una nueva empresa.

H6: Existe una relación de dependencia entre la variable antecedentes de empresarios en la familia y la firme intención de crear una nueva empresa.

La Tabla 11 muestra la prueba estadística para la H ipótesis 6.

La H ipótesis 6 se rechaza para la muestra de Puerto Rico, mientras que se acepta para la muestra de Catalunya. De esta manera, se demuestra que existe una relación de dependencia entre los antecedentes de empresarios en la familia y la seria intención de crear una empresa propia, estadísticamente significativa en un contraste " $\mathrm{x}^{2 \text { " al }}$ nivel de $95 \%$. El porcentaje de estudiantes catalanes con antecedentes de empresarios en la familia que respondió Sí, seriamente (15.0\%) es más elevado que el porcentaje de ellos que no tienen empresarios en la familia (9.6\%), a la vez que es superior al porcentaje total de dicha muestra (12.2\%).

Tabla 11

Tabla de contingencia entre los antecedentes de empresarios en la familia de los estudiantes y la intención de crear una nueva empresa

Por cientos

\begin{tabular}{|c|c|c|c|c|c|}
\hline $\begin{array}{c}\text { CATALUNYA } \\
(\%)\end{array}$ & $\begin{array}{c}\text { No, } \\
\text { nunca }\end{array}$ & $\begin{array}{c}\text { No, pero pienso } \\
\text { integrarme a una } \\
\text { empresa de la familia }\end{array}$ & $\begin{array}{c}\text { Sí, } \\
\text { vagamente }\end{array}$ & $\begin{array}{c}\text { Sí, } \\
\text { seriamente }\end{array}$ & $\begin{array}{l}\text { Si, tengo el firme } \\
\text { propósito de fundar } \\
\text { una empresa }\end{array}$ \\
\hline $\begin{array}{l}\text { Con antecedentes de empresarios } \\
\text { en la familia }\end{array}$ & 21.6 & 6.9 & 52.3 & 15.0 & 4.3 \\
\hline $\begin{array}{l}\text { Sin antecedentes de empresarios } \\
\text { en la familia }\end{array}$ & 32.9 & 1.9 & 51.6 & 9.6 & 4.0 \\
\hline Intencionalidad Total & 27.4 & 4.3 & 52.0 & 12.2 & 4.1 \\
\hline Prueba Estadística & \multicolumn{5}{|c|}{ Valor $\mathrm{x}^{2}=26.250^{* *}$} \\
\hline $\begin{array}{l}\text { Puerto Rico } \\
\quad(\%)\end{array}$ & $\begin{array}{c}\text { No, } \\
\text { nunca }\end{array}$ & $\begin{array}{c}\text { No, pero pienso } \\
\text { integrarme a una } \\
\text { empresa de la familia }\end{array}$ & $\begin{array}{c}\text { Sí, } \\
\text { vagamente }\end{array}$ & $\begin{array}{c}\text { Sí, } \\
\text { seriamente }\end{array}$ & $\begin{array}{l}\text { Sí, tengo el firme } \\
\text { propósito de fundar } \\
\text { una empresa }\end{array}$ \\
\hline $\begin{array}{l}\text { Con antecedentes de empresarios } \\
\text { en la familia }\end{array}$ & 8.3 & 2.4 & 38.3 & 31.6 & 19.4 \\
\hline $\begin{array}{l}\text { Sin antecedentes de empresarios } \\
\text { en la familia }\end{array}$ & 15.0 & 1.2 & 43.7 & 27.5 & 12.6 \\
\hline Intencionalidad Total & 11.3 & 1.9 & 40.8 & 29.8 & 16.4 \\
\hline PRUEBA ESTADÍSTICA & \multicolumn{5}{|c|}{ Valor $\mathrm{x}^{2}=8.2288$} \\
\hline
\end{tabular}

**Sig $<0.05$ 


\section{Conclusiones}

Las principales conclusiones que se desprenden del presente trabajo son las siguientes:

1. El análisis de la información obtenida a partir del cuestionario administrado a los estudiantes universitarios revela que, tanto la muestra catalana como la muestra de Puerto Rico tienen una percepción positiva del deseo de crear una nueva empresa. La mayor parte de los estudiantes, $74.0 \%$ para Catalunya y $92.2 \%$ para Puerto Rico, respondió que consideraba deseable crear una empresa propia.

2. Si se comparan estos resultados con los del estudio realizado en España por Veciana y Genescà ( 1984) se descubre un aumento muy importante en la percepción de deseo para crear una empresa en las dos últimas décadas.

3. Asimismo, comparando los resultados de la presente investigación con los de Veciana (1998a) y U rbano (2003), correspondientesa dos muestras de estu diantes universitarios que estaban cursando la asignatura de creación de empresas, se observa que en este caso la percepción de deseo crear una empresa es aún más favorable con $90.2 \%$ y $89.9 \%$, respectivamente.

4. En cuanto a las respuestas correspondientes a la viabilidad de crear una empresa propia, los resultados son menos favorables. En este sentido, el $66.1 \%$ de la muestra de Catalunya, así como el $53.5 \%$ de la muestra de Puerto Rico considera que es más difícil crear una empresa en la actualidad que en décadas anteriores. En cuanto a la comparación con lostrabajos de Veciana (1998a) y de U rbano (2003) basados en muestras constituidas por estudiantes que estaban cursando la asignatura de creación de empresas, los resultados que se desprenden también apuntan en esta dirección, que es más difícil crear una empresa en la actualidad, a pesar de que la percepción de la viabilidad para la creación de empresas es un poco más positiva.

5. Un elevado porcentaje de estudiantes de ambas muestras respondió tener una vaga intención decrear una nueva empresa ( $51 \%$ para Catalunya y $40.3 \%$ para Puerto Rico) o "seria" ( $12.1 \%$ para Catalunya y $28.7 \%$ para Puerto Rico), a pesar de que sólo un 
porcentaje muy reducido tiene la firme intención de establecerse por cuenta propia ( $4.1 \%$ en el caso de la muestra catalana y $16.1 \%$ en el caso de Puerto Rico).

6. En Catalunya existe una relación de dependencia entre el género de los estudiantes y en deseabilidad, así como entre el género y la intención de fundar una empresa. Son los hombreslos que cuentan con mayores porcentajes de deseo e intención de establecer una empresa.

7. Por otro lado, en la muestra de estudiantes catalanes existe una relación de dependencia entre los antecedentes de empresarios en la familia y la seria inten ción de crear una empresa propia. Estos resultados difieren de los obtenidos en una investigación sobre la población general catalana (Urbano, 2003), donde según la muestra analizada existían relaciones de dependencia estadísticamente significativas entre el género y el deseo, la viabilidad y la intención de fundar una empresa, así como entre los antecedentes de empresarios en la familia y el deseo e intención de fundar una empresa.

8. Por su parte, en el caso de Puerto Rico no se producen relaciones de dependencia en ninguno de los casos considerados en la presente investigación, aunque si se comparan estos resultados con los de un estudio sobre las actitudes hacia la creación de empresas de una muestra de la población general puertorriqueña (Aponte, 2002) , se comprueba que existen relaciones de dependencia estadísticamente significativas entre el género y el deseo, y entre el género y la intención de fundar una empresa propia.

\section{Recomendaciones}

Partiendo de los resultados que evidencian que en ambas muestras de estudiantes bajo estudio la percepción de deseabilidad para crear una empresa propia es más positiva que la viabilidad, se presentan cuatro recomendaciones orientadas, principalmente, a mejorar entre los jóvenes la percepción sobre la viabilidad para la creación de una empresa propia. 
1. Promover el desarrollo de conocimientos y destrezas empresariales en las sociedades catalana y puertorriqueña a través de currículos y programas enfocados a la creación de empresas en las instituciones de educación de todos los niveles de enseñanza, desde la educación primaria hasta la universidad. En el ámbito universitario, sería necesario convertir esta asignatura en una requerida e impartirse en todos los programas de estudiosy con la mayor transversalidad. Dichos currículos deben promover una cultura favorable al empresario.

2. Potenciar el uso de los modelos de referencia de empresarios de éxito como instrumento para mejorar la percepción de vialidad de crear una empresa propia. Si los empresarios potencialestienen la oportunidad de vivir y ver de cerca hechosyejemplos de empresarios de éxito, seguramente aumentará la probabilidad de que decidan crear su propia empresa.

3. Mejorar la cobertura mediática sobre la creación de empresas. Los medios de comunicación deberían dar más importancia a temáticas que potencien el espíritu emprendedor, la iniciativa individual y la creatividad, entre otros.

4. Fomentar entre nuevos empresarios la creación de redes que contribuyan a mejorar las condiciones de éxito en las primeras etapas de vida de la empresa. 


\section{Notas}

${ }^{1}$ Cabe destacar que la mayoría de ellos aplica el enfoque institucional de North para el estudio de los factores que condicionan la creación de empresas en economías en transición (Nee y Young, 1991; Nee, 1992, Litwack, 1993; Westhead, 1995; Brautigam, 1997; Trulsson, 1997; Veciana, 1999; Peng y Shekshnia, 2001; Stein, 2002 y H arper, 2003).

${ }^{2}$ Cabe decir que en el trabajo titulado 'U niversity Students' Attitudes towardsEntrepreneurship: A two Countries Comparison" (Veciana, Aponte, y U rbano, 2005) se analizan más en profundidad las actitudes de los estudiantes universitarios hacia la creación de empresas, tanto en Catalunya como en Puerto Rico, incluyendo también el estudio de los atributos y la percepción del empresario por parte de las muestras de estudiantes consideradas para dichos países.

${ }^{3}$ Entre otros estudios más recientes que abordan esta temática, se pueden mencionar lossiguientes: Brockhaus yH orwitz (1986), Cooper (1986), Scott y Twomey (1988), Scherer, Adams, Carley y Wiebe (1989), M atthews y Moser (1995). 


\section{Bibliografía}

Ajzen, I. (1991): "The Theory of Planned Behavior", Organizational Behavior and H uman Decision Processes, vol.50, pp.179-211.

Aponte, M. (2002): Factores condicionantes de la creación de empresas en Puerto Rico. Un enfoque institucional. Tesis doctoral. Universidad Autónoma de Barcelona.

Brockhaus, R. H ., y Horwitz, P S. (1986): "The Psychology of the Entrepreneur". In D. Sexton \& R. Smilor (Eds.), The Art and Science of Entrepreneurship, pp.25-48.Cambridge, MA: Ballinger.

Collins, O.F., Moore, D. G. yU nwalla, D. B. (1964): The Enterprising Man, MSU Business Studies.

Cooper, A. (1986): "Entrepreneurship and High Technology" In D. Sexton \& R. Smilor (Eds.). The Art and Science of Entrepreneurship, pp. 153-180. Cambridge, MA: Ballinger.

Genescà, E. y Veciana, J. ( 1984): "Actitudes hacia la Creación de Empresas", Información Comercial Española, No.611, pp.147-155, (July).

H arper, D.A. (2003) : Foundations of Entrepreneurship and Economic Development. New York: Routledge.

Katz, J. A. (1992) . A psychological cognitive model of employment status choice. Entrepren eurship Theory and Practice, 17(1), pp. 29-37.

Kolvereid, L. (1996). Prediction of Employment Status Choice Intentions. Entrepren eurship T heory and Practice, 21(1), pp.47-57.

Kourilsky, M.L. yWalstad, W.B. ( 1998): "Entrepreneurship and Female Youth: Knowledge, Attitudes, Gender Differences and Educational Practices", Journal of Business Venturing, 13 (1).

Krueger, N. (1993): "The Impact of Prior Entrepreneurial Exposure on Perceptions", Entrepren eurship Theory and Practice, 18(1), pp.5-21.

Krueger, N. y Brazeal, D. (1994): "Entrepreneurial Potential and Potential Entrepreneurs", Entrepren eurship Theory and Practice, 18(3), pp.91-104.

Matthews, C. H . y Moser, S. B. ( 1995): "Family Background and Gender: Implications for Interest in Small Firm O wnership", Entrepre neurship \& Regional Development, 7(4),365-377. 
North, D. ( 1990) : Institutions, Institutional Changeand Economic Performance. Cambridge U niversity Press.

Reynolds, P. ( 1991): "Sociologyand Entrepreneurship: Concepts and Contributions", Entrepreneurship Theory and Practice, (Winter).

Robinson, P B., Simpson, D. V., Huefner, J. C. y H unt, H. K. (1991): "An Attitude Approach to the Prediction of Entrepreneurship", Entrepren eurship T heory and Practice, 15(4), 13-31.

Scherer, P. D., Adams, J., Carley, S. and Wiebe, F. ( 1989): "Role Model Performance Effects on Development of Entrepreneurial Career Preference", En trepren eurship T heory and Practice, 13( 3) , pp.53-81.

Scott, M. G. y Twomey, D. F. (1988): "The Long-Term Supply of Entrepreneurs: Students Career Aspirations in Relation to Entrepreneurship", Journal of Small Business M anagement, 26(4), pp.5-13.

Shapero, A. (1982): "Social Dimensions of Entrepreneurship", In C. Kent et al. (Eds.), The Encyclopedia of Entrepreneurship, pp.7290. Englewood Cliffs, NJ: Prentice Hall.

U rbano, D. (2003): Factores condicionantres de la creación de empresas en Catalunya. Un enfoque institucional. Tesis doctoral. Universidad Autónoma de Barcelona.

Veciana, J.M. ( 1988): “Empresario y Proceso de Creación de Empresas", R evista Económica de Catalunya, No.8, ( May-August).

Veciana, J.M . (1997): "M itos y Realidad de la Creación de Empresas", Ponencia presentada en los Encuentros de Desarrollo Local y Empleo, en Culleredo, 13-14 N oviembre 1997.

Veciana, J.M. ( 1998a) : “Entrepreneurship Education at the U niversity Level: A Challenge and a Response", Paper presented at the Rencontres de St.Gall 1998, in St. Gallen and Elm, (September 28O ctober 2).

Veciana, J.M. (1998b): "Teoría y Política de la Creación de Empresas", Paper presented at "Jornada dels Economistes", Barcelona.

Veciana, J.M. ( 1999): “Creación de Empresas como Programa de Investigación Científica", R evista Eu ropea de Dirección y Economía de la Empresa, Vol.8, No.3, PP.11-36. 
Veciana, J.M., Aponte, M. y U rbano, D. (1999): “Attitu des Towards Entrepreneurship: Two Countries Comparison", Paper presented at RENT XIII (L ondon, 25-26 November).

Veciana, J.M., Aponte, M. and U rbano, D. (2005) : U niversity students' attitudestowardsentrepreneurship: A two countries comparison. International Entrepren eurship and M anagement Journal. 1 (2) , 165182. 